\title{
The Coin Exchange Problem and the Structure of Cube Tilings
}

\author{
Andrzej P. Kisielewicz and Krzysztof Przesławski \\ Wydział Matematyki, Informatyki i Ekonometrii, Uniwersytet Zielonogórski \\ ul. Z. Szafrana 4a, 65-516 Zielona Góra, Poland
}

A.Kisielewicz@wmie.uz.zgora.pl, K.Przeslawski@wmie.uz.zgora.pl

Submitted: Jul 25, 2011; Accepted: Apr 13, 2012; Published: Jun 6, 2012

\begin{abstract}
It is shown that if $[0,1)^{d}+t, t \in T$, is a unit cube tiling of $\mathbb{R}^{d}$, then for every $x \in T, y \in \mathbb{R}^{d}$, and every positive integer $m$ the number $\left|T \cap\left(x+\mathbb{Z}^{d}\right) \cap\left([0, m)^{d}+y\right)\right|$ is divisible by $m$. Furthermore, by a result of Coppersmith and Steinberger on cyclotomic arrays it is proven that for every finite discrete box $D=D_{1} \times \cdots \times D_{d} \subseteq x+\mathbb{Z}^{d}$ of size $m_{1} \times \cdots \times m_{d}$ the number $|D \cap T|$ is a linear combination of $m_{1}, \ldots, m_{d}$ with non-negative integer coefficients. Several consequences are collected. A generalization is presented.
\end{abstract}

An interest in cube tilings of $\mathbb{R}^{d}$ originated from the following question raised by Hermann Minkowski [23]: Characterize lattices $\Lambda \subset \mathbb{R}^{d}$ such that $[0,1)^{d}+\lambda, \lambda \in \Lambda$, is a cube tiling. Minkowski conjectured that such a lattice $\Lambda$ is of the form $A \mathbb{Z}^{d}$, where $A$ is a lower triangular matrix with ones on the main diagonal. By a simple inductive argument, it is equivalent to showing that $\Lambda$ contains an element of the standard basis. Geometrically, it means that the tiling $[0,1)^{d}+\lambda, \lambda \in \Lambda$, contains a column. This inspired Ott-Heinrich Keller [11] to consider the problem of the existence of columns in arbitrary cube tilings. In [12] he conjectured that starting from dimension 7 there are cube tilings without columns. This has been confirmed in dimension 10 by Jeffrey Lagarias and Peter Shor [18] and in dimension 8 by John Mackey [22]. There are quite a few other papers stemming from Keller's problem [2, 3, 5, 6, 8, 13, 14, 19, 21, 24, 28].

A new stimulus came from Fuglede's conjecture [7]. Several papers appeared at almost the same time where the set determining a cube tiling is characterized as follows: $[0,1)^{d}+t$, $t \in T$, is a cube tiling of $\mathbb{R}^{d}$ if and only if the system of functions $\exp (2 \pi \mathrm{i}\langle t, x\rangle), t \in T$, is an orthonormal basis of $L^{2}\left([0,1]^{d}\right)([9,10,15,17])$.

A reader who seeks an exposition concerning cube tilings is advised to consult $[16,26$, 29].

Let us suppose that $[0,1)^{d}+t, t \in T$, is a cube tiling and that $x \in T$. The simplest unit cube tiling to which the cube $[0,1)^{d}+x$ belongs is $[0,1)^{d}+u, u \in x+\mathbb{Z}^{d}$. We discuss 
here in what way these two tilings are intertwined. To be more specific, we show that there are certain number-theoretic characteristics of the intersection $\left(x+\mathbb{Z}^{d}\right) \cap T$.

A standard block in $\mathbb{R}^{d}$ is a set of the form $X=X_{1} \times \cdots \times X_{d}$, where $X_{i} \in\{[0,1), \mathbb{R}\}$. The translate $X+x$ of a standard block $X \subseteq \mathbb{R}^{d}$ on $x \in \mathbb{R}^{d}$ is called a block in $\mathbb{R}^{d}$. A block which is a translate of $[0,1)^{d}$ is said to be a unit cube. If $F=F_{1} \times \cdots \times F_{d}$ is a block in $\mathbb{R}^{d}$, then the set $N_{F}=\left\{i: F_{i}=\mathbb{R}\right\}$ is called the cosupport of $F$. For future reference, we define the set $\mathbb{Z}_{N_{F}}^{d}$ by the equation

$$
\mathbb{Z}_{N_{F}}^{d}=\left\{\left(k_{1}, \ldots, k_{d}\right) \in \mathbb{Z}^{d}: k_{i}=0, \text { whenever } i \notin N_{F}\right\} .
$$

If $F$ is a block, then its position vector $v=v(F) \in \mathbb{R}^{d}$ is defined as follows

$$
v_{i}= \begin{cases}x_{i}, & \text { if } F_{i}=[0,1)+x_{i} \\ 0, & \text { if } F_{i}=\mathbb{R}\end{cases}
$$

Clearly, $F-v$ is a standard block.

A family $\mathscr{F}$ of disjoint blocks contained in $\mathbb{R}^{d}$ is a block tiling of $\mathbb{R}^{d}$ if $\bigcup \mathscr{F}=\mathbb{R}^{d}$. If $\mathscr{F}$ consists of unit cubes only, then we refer to $\mathscr{F}$ as a cube tiling.

Suppose that there is a unit cube $J$ which belongs to a block tiling $\mathscr{F}$. Then the family of cubes $\left\{(J+k): k \in \mathbb{Z}^{d}\right\} \cap \mathscr{F}$ is called a simple component of $\mathscr{F}$ (containing $J$ ).

THEOREM 1 Suppose that a unit cube $J$ belongs to a block tiling $\mathscr{F}$. Let $\mathscr{C}$ be a simple component of $\mathscr{F}$ containing $J$. Then there is a block tiling $\mathscr{G}$ such that

(1) $v(G)_{i} \in v(J)_{i}+\mathbb{Z}$, for $G \in \mathscr{G}$ and $i \notin N_{G}$;

(2) $J \in \mathscr{G}$ and the simple component of $\mathscr{G}$ containing $J$ equals $\mathscr{C}$.

In particular, $\mathscr{C}$ consists of all unit cubes belonging to $\mathscr{G}$.

Proof. We may assume that $J=[0,1)^{d}$. Let us fix $i \in\{1, \ldots, d\}$ and $\alpha \in(0,1)$. Let $\mathscr{F}^{\alpha}=\left\{F \in \mathscr{F}: v(F)_{i} \in \alpha+\mathbb{Z}\right\}$. If $\mathscr{F}^{\alpha}$ is non-empty, then since $\mathscr{F}$ is a tiling, the family $\mathscr{S}:=\left\{F \in \mathscr{F}^{\alpha}: v(F)_{i}=\alpha\right\}$ is non-empty. For $F \in \mathscr{S}$, let $\bar{F}$ be a block defined so that $\bar{F}_{i}=\mathbb{R}$ and $\bar{F}_{j}=F_{j}$ whenever $j \neq i$. Let $\mathscr{G}_{\alpha}=\{\bar{F}: F \in \mathscr{S}\}$. Again by the fact that $\mathscr{F}$ is a tiling, we deduce that $\bigcup \mathscr{F}^{\alpha}=\bigcup \mathscr{G}_{\alpha}$. If $\mathscr{F}^{\alpha}$ is empty, then we let $\mathscr{G}_{\alpha}$ to be empty. Observe now that the family $\mathscr{F}^{(i)}:=\left(\mathscr{F} \backslash \bigcup_{\alpha \in(0,1)} \mathscr{F}^{\alpha}\right) \cup \bigcup_{\alpha \in(0,1)} \mathscr{G}_{\alpha}$ is a block tiling which has the following properties:

$\diamond$ the simple components of $\mathscr{F}$ and $\mathscr{F}^{(i)}$ containing $J$ coincide;

$\diamond$ if $F \in \mathscr{F}^{(i)}$, then $v(F)_{i} \in \mathbb{Z}$.

Let us define inductively a sequence of block tilings $\left(\mathscr{F}^{[i]}: i=1, \ldots, d\right)$ :

$$
\mathscr{F}^{[1]}=\mathscr{F}^{(1)}, \mathscr{F}^{[2]}=\left(\mathscr{F}^{[1]}\right)^{(2)}, \ldots, \mathscr{F}^{[d]}=\left(\mathscr{F}^{[d-1]}\right)^{(d)} .
$$


It is clear that the simple components of $\mathscr{F}$ and $\mathscr{F}{ }^{[d]}$ containing $J$ coincide and $v(F) \in \mathbb{Z}^{d}$ whenever $F \in \mathscr{F}[d]$. Therefore, it suffices to declare $\mathscr{G}=\mathscr{F}[d]$.

We say that a set $T \subset \mathbb{R}^{d}$ determines a cube tiling $\mathscr{F}$ of $\mathbb{R}^{d}$ if

$$
T=v(\mathscr{F})=\{v(F): F \in \mathscr{F}\}
$$

Let $T$ determine a cube tiling $\mathscr{F}$ and $t \in T$. Then the set $\left(t+\mathbb{Z}^{d}\right) \cap T$ consists of all position vectors of a certain simple component of $\mathscr{F}$. As an immediate consequence we have

THEOREM 2 Given a set $T$ determining a cube tiling of $\mathbb{R}^{d}$. Let $x \in T$ and let $D \subset x+\mathbb{Z}^{d}$ be a finite discrete box with sidelengths equal to $m$; that $i s, D=D_{1} \times \cdots \times D_{d}$ and $m=\left|D_{1}\right|=\cdots=\left|D_{d}\right|$. Then $|T \cap D|$ is divisible by $m$.

Proof. Let $\mathscr{F}$ be the cube tiling determined by $T$. Let $\mathscr{C}$ be the simple component of $\mathscr{F}$ which contains $J=[0,1)^{d}+x$. Let $\mathscr{G}$ be the block tiling of $\mathbb{R}^{d}$ as described in Theorem 1. Since for each $G \in \mathscr{G}$ we have $G=[0,1)^{d}+v(G)+\mathbb{Z}_{N_{G}}^{d}$, it follows that the set

$$
\mathscr{P}=\left\{\left(v(G)+\mathbb{Z}_{N_{G}}^{d}\right) \cap D: G \in \mathscr{G}\right\} \backslash\{\emptyset\}
$$

is a partition of $D$. Observe that $N_{G}$ is non-empty if $G \in \mathscr{G} \backslash \mathscr{C}$. Thus, $H_{G}:=(v(G)+$ $\left.\mathbb{Z}_{N_{G}}^{d}\right) \cap D$ is empty or it is a discrete box such that $H_{i}=D_{i}$, for $i \in N_{G}$. Now, it follows that $\left|H_{G}\right|$ is divisible by $m$. Therefore, the number

$$
|T \cap D|=m^{d}-\sum_{G \in \mathscr{G} \backslash \mathscr{C}}\left|H_{G}\right|
$$

is divisible by $m$.

Let us underline that the sets $D_{1}-x_{1}, \ldots, D_{d}-x_{d}$ are not necessarily intervals of consecutive integers.

Theorem 2 can be generalized to finite discrete boxes of arbitrary size. To this end we shall need a deep result of Coppersmith and Steinberger [4, Theorem 1] concerning the sum of the entries of a cyclotomic array.

Let $\mathbb{N}$ be the set of all positive integers. If $m \in \mathbb{N}$, then $[m]$ denotes, as usual, the initial segment $\{1, \ldots, m\}$. If $\boldsymbol{m}=\left(m_{1}, \ldots, m_{d}\right) \in \mathbb{N}^{d}$, then $[\boldsymbol{m}]:=\left[m_{1}\right] \times \cdots \times\left[m_{d}\right]$. A non-negative integer $n$ is representable by $\boldsymbol{m}$ if there are non-negative integers $n_{1}, \ldots, n_{d}$ such that

$$
n=n_{1} m_{1}+\cdots+n_{d} m_{d} .
$$

In other words, the amount $n$ can be changed using coins of denominations $m_{1}, \ldots, m_{d}$. As a consequence of this interpretation, the problem of representability is often called the coin exchange problem (see e.g. $[1,25]$ ). A line in $[\boldsymbol{m}]$ is any set of the form

$$
\left\{x_{1}\right\} \times \cdots \times\left\{x_{s-1}\right\} \times\left[m_{s}\right] \times\left\{x_{s+1}\right\} \times \cdots \times\left\{x_{d}\right\},
$$

where $s \in[d]$, and $x_{i} \in\left[m_{i}\right]$. A subset $Q$ of $[\boldsymbol{m}]$ is said to be complementable by lines if its complement $[\boldsymbol{m}] \backslash Q$ can be represented as a union of disjoint lines. A characteristic 
function $f:[\boldsymbol{m}] \rightarrow\{0,1\}$ is called a fiber if its support is a line. Following Steinberger [27], a mapping $A:[\boldsymbol{m}] \rightarrow \mathbb{Z}$ is said to be a cyclotomic array if it is a linear combination of fibers with integer coefficients. The result of Coppersmith and Steinberger reads that the sum $\sum_{\boldsymbol{x} \in[\boldsymbol{m}]} A(\boldsymbol{x})$ is representable by $\boldsymbol{m}$ if $A$ is a nonnegative cyclotomic array. As an immediate consequence we have

Proposition 3 For each $\boldsymbol{m} \in \mathbb{N}^{d}$, if $Q \subseteq[\boldsymbol{m}]$ is complementable by lines, then $|Q|$ is representable by $\boldsymbol{m}$.

TheOREM 4 Given a set $T$ determining a cube tiling of $\mathbb{R}^{d}$. Let $x \in T$ and let $D \subset$ $x+\mathbb{Z}^{d}$ be a finite discrete box of size $m_{1} \times \cdots \times m_{d}$. Then $|T \cap D|$ is representable by $\boldsymbol{m}=\left(m_{1}, \ldots, m_{d}\right)$.

Proof. Since the proof is a slight modification of the proof of Theorem 2, the notation used there is preserved. Let us pick a system of bijections $\varphi_{i}: D_{i} \rightarrow\left[m_{i}\right], i \in[d]$, and define a bijection $\varphi: D \rightarrow[\boldsymbol{m}]$ by the formula $\varphi=\varphi_{1} \times \cdots \times \varphi_{d}$. Let $Q=\varphi(T \cap D)$. The set $[\boldsymbol{m}] \backslash Q$ is a disjoint union of the sets $\varphi\left(H_{G}\right), G \in \mathscr{G} \backslash \mathscr{C}$. By the definition of $\varphi$ and the fact that each $H_{G}$ is a box with side $D_{i}$, for some $i$, it follows that each $\varphi\left(H_{G}\right)$ is a disjoint union of lines. Now, as $Q$ satisfies the assumption of Proposition $3,|Q|$ is representable by $\boldsymbol{m}$.

Corollary 5 Given a set $T$ determining a cube tiling of $\mathbb{R}^{d}$. Let $x \in T$ and let $B$ be a half-open box of size $m_{1} \times \cdots \times m_{d}$, that is, there is $y \in \mathbb{R}^{d}$ such that $B=\left[y_{1}, y_{1}+m_{1}\right) \times$ $\cdots \times\left[y_{d}, y_{d}+m_{d}\right)$. Then $\left|T \cap\left(x+\mathbb{Z}^{d}\right) \cap B\right|$ is representable by $\boldsymbol{m}=\left(m_{1}, \ldots, m_{d}\right)$.

Proof. Define $D:=\left(x+\mathbb{Z}^{d}\right) \cap B$. As $D$ is a discrete box of size $m_{1} \times \cdots \times m_{d}$, Theorem 4 applies to reach the conclusion.

Corollary 6 Given a set $T$ determining a cube tiling of $\mathbb{R}^{d}$. Let $x \in T$ and let $B$ be a closed box of size $m_{1} \times \cdots \times m_{d}$, that is, there is $y \in \mathbb{R}^{d}$ such that $B=\left[y_{1}, y_{1}+m_{1}\right] \times \cdots \times$ $\left[y_{d}, y_{d}+m_{d}\right]$. Then the cardinality of the set $Q:=\left\{t \in T \cap\left(x+\mathbb{Z}^{d}\right):\left(t+[0,1)^{d}\right) \cap B \neq \emptyset\right\}$ is representable by $\left(m_{1}+1, \ldots, m_{d}+1\right)$.

Proof. Define

$$
D:=\left(x+\mathbb{Z}^{d}\right) \cap\left(\left(y_{1}-1, y_{1}+m_{1}\right] \times \cdots \times\left(y_{d}-1, y_{d}+m_{d}\right]\right)
$$

$D$ is a discrete box of size $\left(m_{1}+1\right) \times \cdots \times\left(m_{d}+1\right)$. Moreover, $Q=T \cap D$. Again, Theorem 4 leads to the conclusion.

Let $\boldsymbol{m}=\left(m_{1}, \ldots, m_{d}\right) \in \mathbb{N}^{d}$ and let $T$ determine a cube tiling of $\mathbb{R}^{d}$. This tiling is said to be $\boldsymbol{m}$-periodic if for every vector of the standard basis $e_{1}=(1,0, \ldots, 0), \ldots, e_{d}=$ $(0, \ldots, 0,1)$ one has

$$
T+m_{i} e_{i}=T
$$


We define the (flat) torus $\mathbb{T}_{\boldsymbol{m}}^{d}$ to be the set $\left[0, m_{1}\right) \times \cdots \times\left[0, m_{d}\right)$ with addition $\bmod \boldsymbol{m}$ :

$$
x \oplus y:=\left(\left(x_{1}+y_{1}\right) \bmod m_{1}, \ldots,\left(x_{d}+y_{d}\right) \bmod m_{d}\right) .
$$

We can extend the notion of a cube so that it will apply to flat tori: Cubes in $\mathbb{T}_{m}^{d}$ are the sets of the form $[0,1)^{d} \oplus t$, where $t \in \mathbb{T}_{m}^{d}$. It is clear that we can speak about cube tilings of $\mathbb{T}_{m}^{d}$ and that there is a canonical 'one-to-one' correspondence between these tilings and the $\boldsymbol{m}$-periodic tilings of $\mathbb{R}^{d}$. We say that $T \subset \mathbb{T}_{\boldsymbol{m}}^{d}$ determines a cube tiling of $\mathbb{T}_{\boldsymbol{m}}^{d}$ if $[0,1)^{d} \oplus t, t \in T$ is a tiling. Let $\mathbb{Z}_{m}^{d}=\mathbb{Z}_{m_{1}} \times \cdots \times \mathbb{Z}_{m_{d}}$. By the analogy to cube tilings of $\mathbb{R}^{d}$, every set $T \cap\left(x \oplus \mathbb{Z}_{\boldsymbol{m}}^{d}\right)$, where $x \in T$, determines a simple component of the tiling $[0,1)^{d} \oplus t, t \in T$. As a consequence of Corollary 5 we have

TheOREM 7 If $T$ determines a cube tiling of a torus $\mathbb{T}_{m}^{d}$ and $S$ determines a simple component of this tiling, then $|S|$ is $\boldsymbol{m}$ representable.

Our main result (Theorem 4) holds in a more general setting. We need some additional terminology in order to formulate such a generalization (compare [21]).

Let $V$ be a non-empty set. A family $\mathscr{V} \subseteq 2^{V} \backslash\{\emptyset, V\}$ is distinctive if for every $A \in \mathscr{V}$, there is a unique partition $\mathscr{C}_{A} \subseteq \mathscr{V}$ of $V$ such that $A \in \mathscr{C}_{A}$.

The family of all unit segments $[0,1)+x, x \in \mathbb{R}$, is distinctive while the family of unit squares $[0,1)^{2}+x, x \in \mathbb{R}^{2}$, is not. On the other hand, the family of all translates of a regular hexagon in $\mathbb{R}^{2}$ with three consecutive sides removed is distinctive.

Let $X$ be the Cartesian product of sets $X_{i}, i \in[d]$. A non-empty subset $A$ of $X$ is called a box (in $X$ ) if $A=A_{1} \times \cdots \times A_{d}$ and $A_{i} \subseteq X_{i}$ for each $i \in[d]$.

Let $\mathscr{X}_{i} \subseteq 2^{X_{i}} \backslash\left\{\emptyset, X_{i}\right\}, i \in[d]$. We denote by $\mathscr{X}=\mathscr{X}_{1} \otimes \cdots \otimes \mathscr{X}_{d}$ the family of all boxes $A \subseteq X=X_{1} \times \cdots \times X_{d}$ such that $A_{i} \in \mathscr{X}_{i}$ for every $i \in[d]$. If each $\mathscr{X}_{i}$ is distinctive, then $\mathscr{X}$ is called a free family of boxes on $X$. Let us fix $A \in \mathscr{X}$. Then $\mathscr{C}_{A}=\mathscr{C}_{A_{1}} \otimes \cdots \otimes \mathscr{C}_{A_{d}} \subseteq \mathscr{X}$ is a partition of $X$. We refer to $\mathscr{C}_{A}$ as a simple partition of $X$ (determined by $A$ ).

The announced generalization can be proved along the same lines as Theorem 4.

TheOREM 8 Let $\mathscr{X}$ be a free family of boxes on $X=X_{1} \times \cdots \times X_{d}$. Let $\mathscr{K} \subseteq \mathscr{X}$ be a partition of $X$, and $A \in \mathscr{K}$. Let $\mathscr{D}$ be a finite discrete box of size $\boldsymbol{m} \in \mathbb{N}^{d}$ contained in the simple partition $\mathscr{C}_{A}$, that $i s, \mathscr{D}=\mathscr{D}_{1} \otimes \cdots \otimes \mathscr{D}_{d}, \mathscr{D}_{i} \subseteq \mathscr{C}_{A_{i}}$ and $\left|\mathscr{D}_{i}\right|=m_{i}$, for $i \in[d]$. Then $|\mathscr{D} \cap \mathscr{K}|$ is representable by $\boldsymbol{m}$.

Acknowledgments. We thank Mihalis Kolountzakis who brought our attention to the connection between Proposition 3 that had been conjectured in a previous version of this note and a paper of Tsit Yuen Lam and Ka Hin Leung on vanishing sums of roots of unity [20] which eventually led us to the result of Coppersmith and Steinberger on cyclotomic arrays.

We also wish to express our thanks to an anonymous referee for valuable comments. 


\section{References}

[1] M. Beck and S. Robins, Computing the Continuous Discretely, Springer, New York, 2007.

[2] K. Corrádi and S. Szabó, Cube tiling and covering a complete graph, Discrete Math. 85 (1990), 319-321.

[3] K. Corrádi and S. Szabó, A combinatorial approach for Keller's conjecture, Period. Math. Hungar. 21 (1990), 95-100.

[4] D. Coppersmith and J. Steinberger, On the Entry Sum of Cyclotomic Arrays, INTEGERS 6 (2006), \# A26, pp 28.

[5] M. Dutour Sikirić and Y. Itoh, Combinatorial cube packings in the cube and the torus, European J. Combin. 31 (2010), 517-534.

[6] M. Dutour Sikirić, Y. Itoh and A. Poyarkov, Cube packings, second moment and holes, European J. Combin. 28 (2007), 715-725.

[7] B. Fuglede, Commuting self-adjoint partial differential operators and a group theoretic problem, J. Funct. Anal. 16 (1974), 101-121.

[8] G. Hajós, Über einfache und mehrfache Bedeckung des n-dimensionalen Raumes mit einem Würfelgitter, Math. Z. 47 (1941), 427-467.

[9] A. Iosevich and S. Pedersen, Spectral and tiling properties of the unit cube, Inter. Math. Res. Notices 16 (1998), 819-828.

[10] P. Jorgensen and S. Pedersen, Spectral pairs in Cartesian coordinates, J. Fourier Anal. Appl. 5 (1999), 285-302.

[11] O. H. Keller, Über die lückenlose Erfülung des Raumes Würfeln, J. Reine Angew. Math. 163 (1930), 231-248.

[12] O. H. Keller, Ein Satz über die lückenlose Erfüllung des 5- und 6-dimensionalen Raumes mit Würfeln, J. Reine Angew. Math. 177 (1937), 61-64.

[13] A. P. Kisielewicz, K. Przesławski, Polyboxes, cube tilings and rigidity, Discrete Comput. Geom. 40 (2008), 1-30.

[14] M. N. Kolountzakis, Lattice tilings by cubes: whole, notched and extended, Electron. J. Combin. 5 (1998), \#R14, pp 11.

[15] M. N. Kolountzakis, Packing, tiling, orthogonality and completeness, Bull. London Math. Soc. 32 (2000), 589-599.

[16] M. N. Kolountzakis, The study of translational tiling with Fourier Analysis, Fourier Analysis and Convexity, 131-187, Appl. Numer. Harmon. Anal., Birkhäuser Boston, Boston, MA, 2004.

[17] J. C. Lagarias, J. A. Reeds and Y. Wang, Orthonormal bases of exponentials for the n-cube, Duke. Math. J. 103 (2000), 25-37.

[18] J. C. Lagarias and P. W. Shor, Keller's cube-tiling conjecture is false in high dimensions, Bull. Amer. Math. Soc. 27 (1992), 279-287. 
[19] J. C. Lagarias and P. W. Shor, Cube tilings and nonlinear codes, Discrete Comput. Geom. 11 (1994), 359-391.

[20] T.Y. Lam, K.H. Leung, On vanishing sums of roots of unity, J. Algebra 224 no. 1 (2000), 91-109.

[21] M. Łysakowska and K. Przesławski, Keller's conjecture on the existence of columns in cube tilings of $\mathbb{R}^{n}$, Advances in Geometry 12 (2012), 329-352.

[22] J. Mackey, A cube tiling of dimension eight with no facesharing, Discrete Comput. Geom. 28 (2002), 275-279.

[23] H. Minkowski, Diophantische Approximationen, Teubner, Leipzig, 1907.

[24] O. Perron, Über lückenlose Ausfüllung des $n$-dimensionalen Raumes durch kongruente Würfel, Math. Z. 46 (1940), 1-26.

[25] J. L. Ramírez Alfonsín, The Diophantine Frobenius Problem, Oxford University Press, New York, 2005.

[26] S. K. Stein and S. Szabó, Algebra and Tiling: Homomorphisms in the Service of Geometry, American Mathematical Association, Washington, 1994.

[27] J. Steinberger, Minimal vanishing sums of roots of unity with large coefficients, Proc. London Math. Soc. 97 (2008), 689-717.

[28] S. Szabó, Cube tilings as contributions of algebra to geometry, Beiträge Algebra Geom. 34 (1993), 63-75.

[29] Ch. Zong, The Cube: A Window to Convex and Discrete Geometry, Cambridge Tracts in Mathematics (168), 2006. 